\title{
An Optical Pulse-Width Modulation Generator Using a Single-Mode Fabry-Pérot Laser Diode
}

\author{
Quoc-Hoai Tran ${ }^{\dagger}$, Bikash Nakarmi, and Yong Hyub Won* \\ Department of Electrical Engineering (EE), Korea Advanced Institute of Science and Technology (KAIST), \\ 291 Daehak-ro, Yuseong-gu, Daejeon 305-701, Korea
}

(Received February 12, 2015 : revised May 18, 2015 : accepted May 19, 2015)

\begin{abstract}
We have proposed and experimentally verified a pulse-width modulation (PWM) generator which directly generated a PWM signal in the optical domain. Output waveforms were clear at the repetition rate of $16 \mathrm{MHz}$; the duty cycle (DC) was from $14.7 \%$ to $72.1 \%$; and the DC-control resolution was about 4.399 $\% / \mathrm{dB}$. The PWM generator's operation principle is based on the injection-locking property of a single-mode Fabry-Pérot laser diode (SMFP-LD). The SMFP-LD, which has a self-locked mode wavelength at $\lambda_{\text {PWM }}$, was used to detect the power of the injection-locking signal (optical analog input). If the analog input power is high, the SMFP-LD is locked to the wavelength of the input signal $\lambda_{\mathrm{a}}$ and there is no output after an optical bandpass filter (OBF). If the analog input power is low, the SMFP-LD is unlocked and there is output signal at $\lambda_{\mathrm{PWM}}$ after the OBF. Thus, the SMFP-LD plus the OBF provide digital output for an analog input. The DC of the output PWM signal can be controlled by tuning the power of the analog input.
\end{abstract}

Keywords: Optical pulse width modulation, Single mode FP-LD, Injection-locking

OCIS codes : (140.3520) Lasers, injection-locked; (140.3570) Lasers, single-mode; (250.0250) Optoelectronics

\section{INTRODUCTION}

Pulse-width modulation (PWM) is a well-known technique in the electrical domain and is used in several applications, such as telecommunications, power delivery and speed control, voltage regulation, inverters, and audio effects and amplification. In telecommunications, widths of pulses are used to specify data values, which are encoded at one end and decoded at the other [1]. PWM can be used to control the amount of power delivered to a load without incurring the losses that would result from linear power delivery by resistive means [2]. In efficient voltage regulators, PWM is used to switch voltage to the load with an appropriate DC to approximate an output voltage at the desired level $[3,4]$. PWM is also used in sound (music) synthesis, in particular subtractive synthesis, as it gives a sound effect similar to a chorus, or slightly detuned oscillators played together [5]. Since electrical PWM is not suitable for applications where an electrically isolated amplifier is required (e.g. in medical electronics $[6,7])$, nor in applications where a large common-mode signal is considered an important factor of disturbance in control (such as in the open/closed period of a Light-Triggered Thyristor (LTT) in high-voltage direct current (HVDC) conversion $[8,9])$. Several schemes have been proposed so far for producing optical PWM signals [6, 10, 11]; however, none of them can directly generate an optical PWM signal in the optical domain. The common way to get an optical PWM signal in the schemes of $[6,10,11]$ is to produce an electrical PWM signal first, then convert it into an optical PWM signal using an $\mathrm{O} / \mathrm{E}$ converter. We have previously proposed an optical PWM generator (OPWMG) scheme using a SMFP-LD [12] in [13], in which analog signal power is kept constant while additional beam power (of the same wavelength as the analog input) is varied to control the DC. Since the analog input and additional beam have the same wavelength, the control of DC is difficult; hence this approach has the drawback of imprecise control of the output DC. In this paper, we propose another OPWMG scheme that has a larger DC range (14.7\%-72.1\%) and more precise control of the output DC, compared to that of the scheme proposed in [13]. In addition, a duty cycle versus analog input power $\left(\mathrm{DC}-\mathrm{vs}-\mathrm{P}_{\lambda_{\mathrm{a}}}\right)$ curve with $\mathrm{DC}$ control

\section{※Corresponding authors: *yhwon@kaist.ac.kr, ${ }^{\dagger}$ hoaitq@kaist.ac.kr}

Color versions of one or more of the figures in this paper are available online. 
resolution of about $4.399 \% / \mathrm{dB}$ was obtained. In this proposed scheme, the analog input power $\mathrm{P}_{\lambda_{\mathrm{a}}}$ is varied using a variable optical attenuator (VOA) to control the DC, and the assisting beam, which has the same wavelength as that of the SMFPLD's self-locked mode $\left(\lambda_{\text {PWM }}\right)$, has constant power $\mathrm{P}_{\lambda \text { asst. }}$ The assisting beam in this paper is used to assist the SMFP-LD's self-locked mode to quickly recover its original (unlocked) state, when $\mathrm{P}_{\lambda \mathrm{a}}$ is lower than the release power level, $\mathrm{P}_{\mathrm{RL}}$. With the scheme proposed here, we experimentally found that the output waveform is clearer and the DC of the output PWM signal is easier to control.

\section{OPERATION PRINCIPLE}

A simple electrical PWM generator is composed of three functional parts [13]: an analog input, a reference, and a comparator. The comparator provides high output (ON) when the input value is less than the reference, and low output (OFF) when the input value is greater than or equal to the reference. Hence, based on the inequality between the analog input (typically a saw-tooth, ramp, triangle, or sinusoidal wave) and the reference, the comparator will provide a square wave as output (digital output) with tunable ON/OFF duration. Figure 1 shows the block diagram of the proposed OPWMG, where the output PWM signal is taken after the optical bandpass filter (OBF) at wavelength $\lambda_{\mathrm{PWM}}$. The SMFPLD used in our experiment was specially designed and developed in our laboratory to have a dominant self-locked mode with a high side-mode-suppression ratio [13]. To shorten the SMFP-LD's recovery time after being injection-locked by the analog signal from an optical analog signal generator, the assisting light beam's wavelength $\lambda_{\text {asst }}$ is set to be the same as that of the SMFP-LD's dominant self-locked mode $\left(\lambda_{\text {ast }}=\lambda_{\text {PWM }}\right)$. In this proposed scheme, the assisting beam power $\mathrm{P}_{\lambda \text { asst }}$ is kept constant; the assisting beam is used to help the SMFP-LD's self-locked mode to recover the unlocked state quickly, which can yield better output waveforms. The SMFP-LD in the proposed scheme has the property of bistability [14], which is explained as follow: When the SMFP-LD is injected with appropriate power from an external light beam

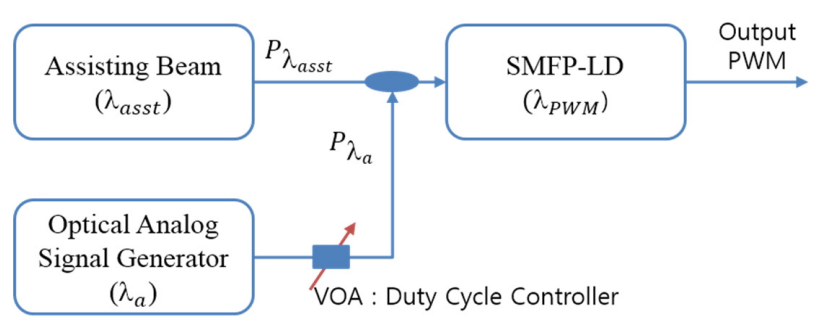

FIG. 1. Block diagram of the OPWMG where $\lambda_{\mathrm{a}}$ is the wavelength of the optical signal generator, $\lambda_{\text {asst }}$ the wavelength of the assisting beam, $\lambda_{\mathrm{PWM}}$ the self-lock mode's wavelength of the SMFP-LD (which carries the PWM signals), VOA the Variable Optical Attenuator, and PWM is Pulse Width Modulation. at a wavelength close enough to one of the SMFP-LD's side modes, it is fully injection-locked, and all of its side modes are suppressed. That power level is called full-lock power $\left(\mathrm{P}_{\mathrm{FL}}\right)$. The SMFP-LD's locking state is released when the injected power is decreased to a level called release power $\left(\mathrm{P}_{\mathrm{RL}}\right)$. The SMFP-LD works as a comparator; both $\mathrm{P}_{\mathrm{FL}}$ and $\mathrm{P}_{\mathrm{RL}}$ were used as references, and $\mathrm{P}_{\lambda_{\mathrm{a}}}$ is used to control the $\mathrm{DC}$.

According to the injection-locking and bistability properties of the SMFP-LD, the locking duration can be varied when the power differences $\left(\mathrm{P}_{\lambda_{a}}-\mathrm{P}_{\mathrm{FL}}\right.$ and $\left.\mathrm{P}_{\lambda_{a}}-\mathrm{P}_{\mathrm{RL}}\right)$ are varied. In other words, the DC can be varied as the change of $\mathrm{P}_{\lambda \mathrm{a}}$ compared to $\mathrm{P}_{\mathrm{RL}}$ and $\mathrm{P}_{\mathrm{FL}}$. In this proposed scheme, $\mathrm{P}_{\lambda_{\mathrm{a}}}$ is varied to control the relative difference between $P_{\lambda a}$ and $\mathrm{P}_{\mathrm{F} L} / \mathrm{P}_{\mathrm{RL}}$. It is noted that when $\lambda_{\mathrm{a}}, \lambda_{\text {asst }}$, and $\mathrm{P}_{\lambda \text { asst }}$ are constant, only power from the analog input contributes to the control of PWM's DC. In fact, $\mathrm{P}_{\mathrm{RL}}$ and $\mathrm{P}_{\mathrm{FL}}$ can be changed, depending on the wavelength detuning of $\lambda_{\mathrm{a}}$ to the corresponding side mode of the SMFP-LD; however, in this paper we only focus on one specific wavelength detuning.

Figure 2 shows the relationship between $\mathrm{P}_{\lambda_{\mathrm{a}}}$ and the output power, where the red dashed and black solid lines represent respectively the states of the analog input and output PWM signals before and after increasing $\mathrm{P}_{\lambda_{\mathrm{a}}}$. $\mathrm{P}_{\lambda_{\mathrm{a}}}$ is controlled by the VOA after the MOD, as shown in Fig. 3. When $\mathrm{P}_{\lambda_{\mathrm{a}}}$ is increased, more of the sinusoidal wave is above the locking power, and the locking duration is increased, or, in other words, the $\mathrm{DC}$ is decreased $\left(\mathrm{DC}_{2}<\mathrm{DC}_{1}\right)$. It is noted that the period is unchanged, if the analog input period $(\mathrm{T})$ is constant $\left(\mathrm{T}_{2}=\mathrm{T}_{1}=\mathrm{T}\right)$. In conclusion, optical PWM signals can be generated in the optical domain, and the PWM's DC can be controlled by varying the power of the analog input.

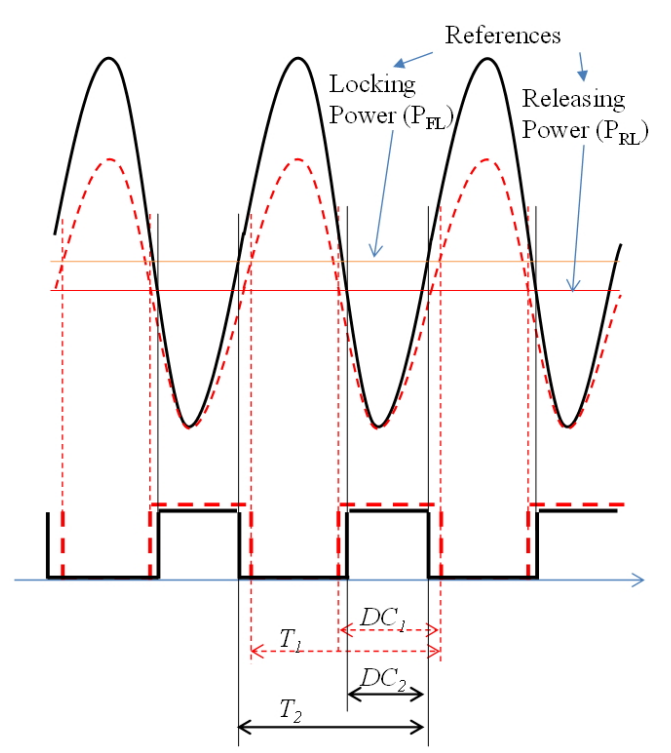

FIG. 2. Generated PWM before (red dashed line) and after (solid black line) increased input power, for the case where the input source is a sinusoidal wave. $\mathrm{DC}_{1}, \mathrm{~T}_{1}, \mathrm{DC}_{2}$, and $\mathrm{T}_{2}$ are respectively the duty cycle and period of the output PWM generator before and after the analog input power is increased. 


\section{EXPERIMENTAL SETUP AND RESULTS}

Figure 3 shows the experimental setup of the OPWMG where the threshold current, bias current, and temperature of the SMFP-LD are $11 \mathrm{~mA}, 19 \mathrm{~mA}$, and $25.3^{\circ} \mathrm{C}$ respectively. Under these working conditions the SMFP-LD has dominant modes at $\lambda_{\text {PWM }}=1546.96 \mathrm{~nm}$. The optical analog input has a wavelength of $1550.4 \mathrm{~nm}$, corresponding to the SMFP-LD's side mode wavelength of $1550.28 \mathrm{~nm}$ (wavelength detuning $\Delta \lambda=1550.4-1550.28=0.12 \mathrm{~nm})$. The assisting beam's wavelength $\lambda_{\text {asst }}$ was set to be the same as that of the SMFP-LD's self-locked mode $\left(\lambda_{\text {asst }}=\lambda_{\text {PWM }}=1546.96 \mathrm{~nm}\right)$, and its lasing power was fixed at about $-10.70 \mathrm{dBm}$ (measured before the optical circulator). When the assisting beam was turned on, the SMFP-LD was fully locked for $\mathrm{P}_{\lambda_{a}} \geq-5.77 \mathrm{dBm}$, and the SMFP-LD's full-lock state was released for $\mathrm{P}_{\lambda_{\mathrm{a}}} \leq$ $-6.68 \mathrm{dBm}$. Actually, the locking state was not fully released even when the input power was less than $-6.68 \mathrm{dBm}$; we still observed the weak-lock phenomenon, where the self-locked mode power was reduced but not fully eliminated. The polarization controller $\mathrm{PC}_{1}$ is used to minimize the polarizationdependent loss in the Mach-Zehnder modulator (MOD), and $\mathrm{PC}_{2}$ and $\mathrm{PC}_{3}$ are used to maximize the TE optical powers

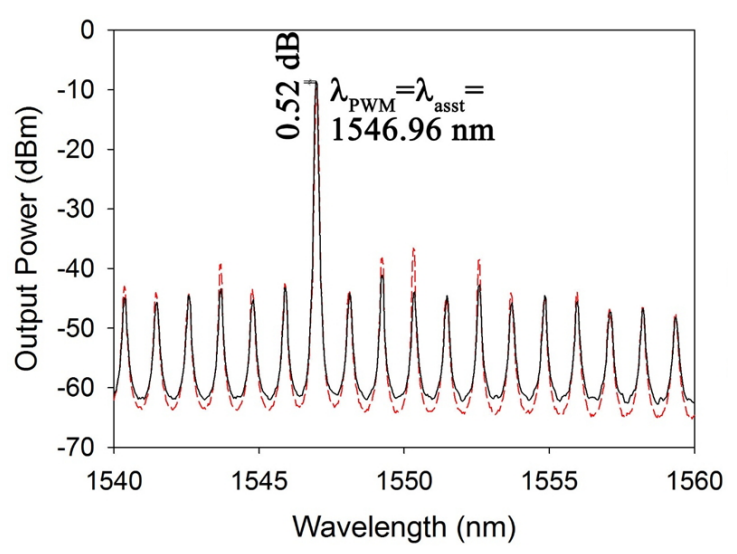

(a) $\mathrm{W} /$ and w/o assisting beam

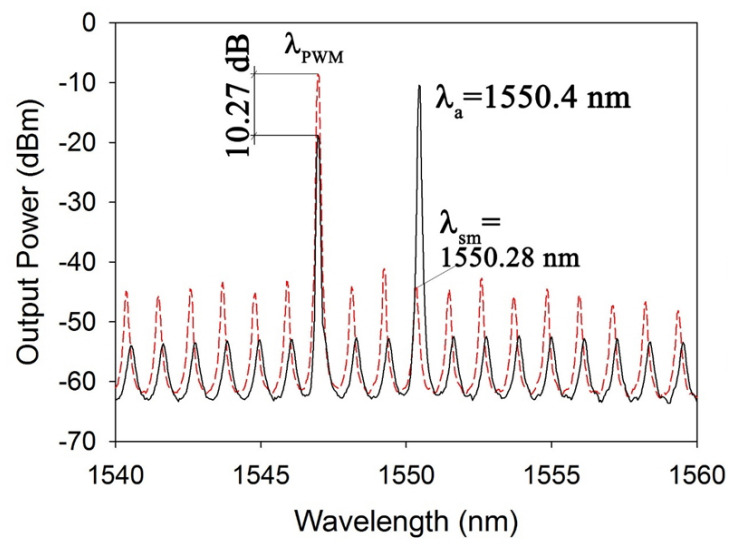

(c) $\mathrm{P}_{\lambda_{\mathrm{a}}}=-4.48 \mathrm{dBm}$ that are injected into the SMFP-LD. Due to the injectionlocking property of the FP-LD, the more TE power that is injected into the SMFP-LD, the greater the suppression of its side modes and the higher the power at the injected mode.

Figure 4 presents the spectra at the output of the SMFP-LD before the OBF, where in Fig. 4(a) the red dashed line

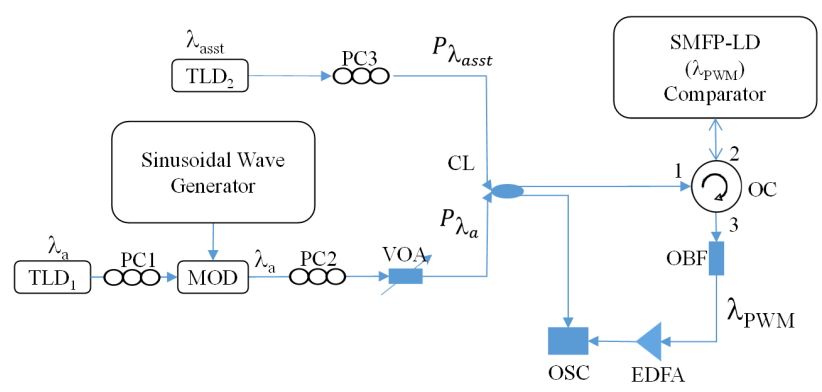

FIG. 3. Experimental setup of the OPWMG, where PC is the Polarization Controller, TLD the Tunable Laser Diode, MOD the Electro-absorption Optical Modulator, VOA the Variable Optical Attenuator, OC the Optical Circulator, CL the Optical Coupler, OBF the Optical Bandpass Filter, OSC the Oscilloscope, and EDFA is the Erbium-Doped Fiber Amplifier.

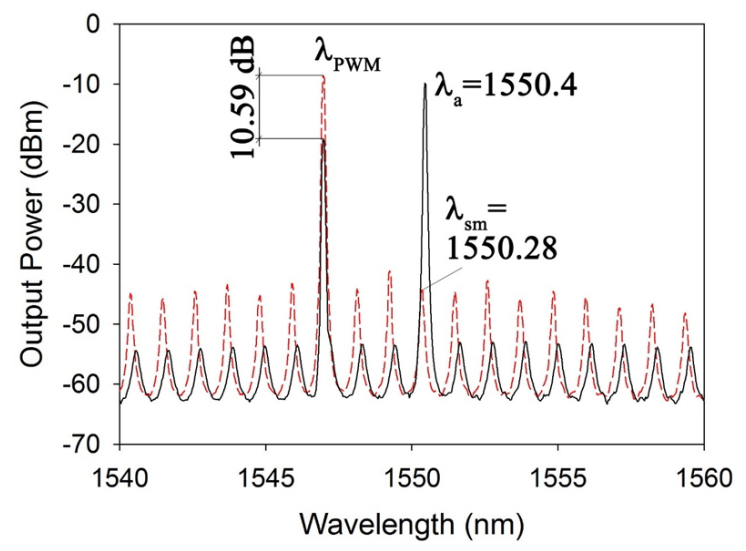

(b) $\mathrm{P}_{\lambda \mathrm{a}}=-2.48 \mathrm{dBm}$

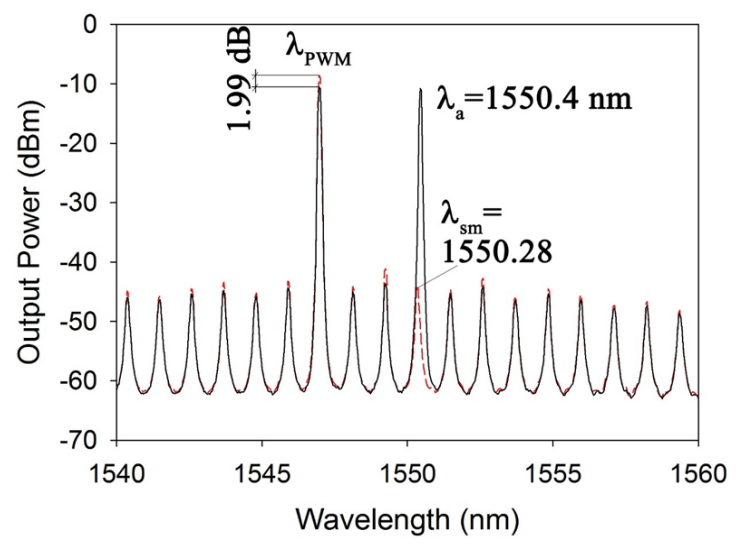

(d) $\mathrm{P}_{\lambda \mathrm{a}}=-7.48 \mathrm{dBm}$

FIG. 4. Output spectra of the OPWMG at the output of the SMFP-LD and before the OBF, (a) without the assisting beam and analog input (red dashed line) and with only the assisting beam (black solid line), and with both assisting beam and analog input in the cases (b) $P \lambda a=-2.48$, (c) -4.48 , and (d) $-7.48 \mathrm{dBm}$, respectively. 


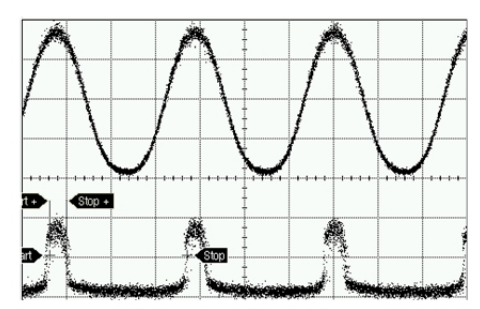

(a) $-2.48 \mathrm{dBm}, 14.7 \%$

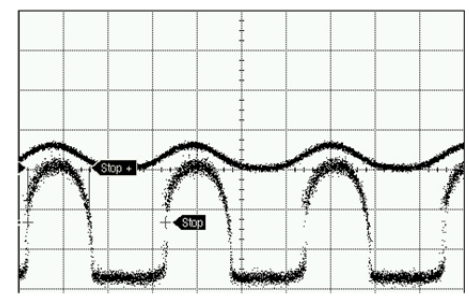

(d) $-10.48 \mathrm{dBm}, 44.8 \%$

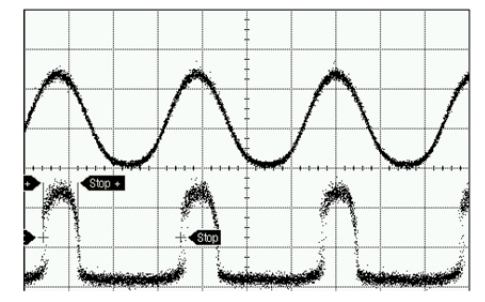

(b) $-4.48 \mathrm{dBm}, 25.0 \%$

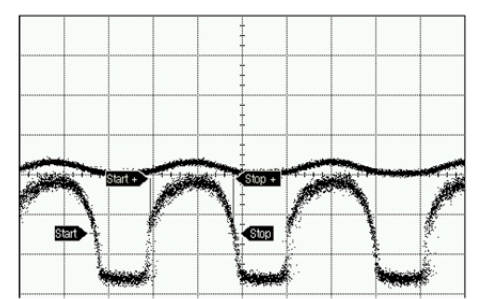

(e) $-13.48 \mathrm{dBm}, 59.8 \%$

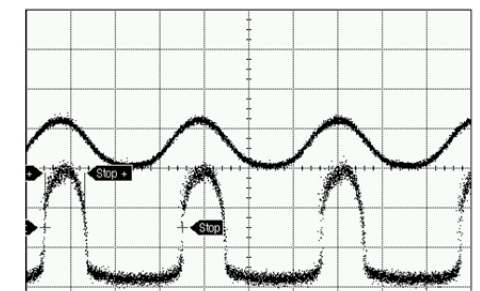

(c) $-7.48 \mathrm{dBm}, 29.8 \%$

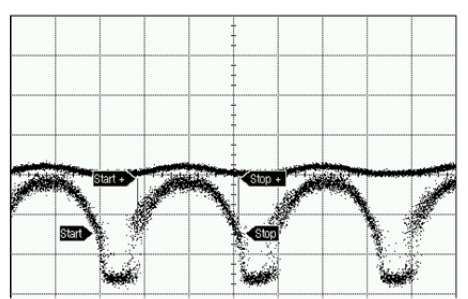

(f) $-15.48 \mathrm{dBm}, 72.1 \%$

FIG. 5. Optical input and output waveforms for various cases of analog input power $\mathrm{P}_{\lambda a}$ and output duty cycle $D C$. The sinusoidal source works at a frequency of $16 \mathrm{MHz}$ and the oscilloscope has a time resolution of $20 \mathrm{~ns} / \mathrm{div}$.

represents the SMFP-LD spectrum only (assisting beam and analog input are turned off) and the black solid line represents the SMFP-LD spectrum when only the assisting beam is turned on. In this figure we can see the weak-lock phenomenon, where side-mode powers are reduced a little but not fully suppressed. In Figs. 4(b), 4(c), and 4(d) the red dashed line represents the spectra of the SMFP-LD when only the assisting beam is $\mathrm{ON}$, and the black solid line represents the spectra of the SMFP-LD before the OBF when both assisting beam and optical analog input are $\mathrm{ON}\left(\mathrm{P}_{\lambda_{\mathrm{a}}}=-2.48,-4.48\right.$, and -7.48 $\mathrm{dBm}$ respectively). We can see the full-lock phenomena in Figs. 4(b) and 4(c), where all side modes of the SMFP-LD are deeply suppressed because $\mathrm{P}_{\lambda_{\mathrm{a}}}(-2.48$ and $-4.48 \mathrm{dBm})$ is above the full-lock power level $\mathrm{P}_{\mathrm{FL}}=-5.77 \mathrm{dBm}$, whereas the full-lock state is released (all side modes are recovered) in Fig. 4(d) because $P_{\lambda a}=-7.48 \mathrm{dBm}$, which is below the release power level $\mathrm{P}_{\mathrm{RL}}=-6.68 \mathrm{dBm}$.

Figure 5 shows optical sinusoidal waveforms of the analog input signal taken before the optical circulator (OC), and the output optical PWM signal taken after the EDFA. The analog optical sinusoidal source is operated at a frequency of $16 \mathrm{MHz}$, and the oscilloscope has a time resolution of $20 \mathrm{~ns} /$ div. In Fig. 5, the input and output waveforms appear to be almost in phase, contradicting their working principle, which is out of phase, as shown in Fig. 2. The reason is that the time difference between the two light paths traveled to the oscilloscope (OSC) is about one period, which is equivalent to $12.5 \mathrm{~m}$ of optical fiber. The output PWM has DCs of $14.7 \%, 25.0 \%, 29.8 \%, 48.8 \%, 59.8 \%$, and $72.1 \%$, corresponding to $\mathrm{P}_{\lambda_{\mathrm{a}}}=-2.48,-4.48,-7.48,-10.48,-13.48$, and $-15.48 \mathrm{dBm}$ respectively.

By changing the analog sinusoidal source power in intervals of about $0.5 \mathrm{~dB}$, we measured the $\mathrm{DC}$ of the proposed OPWMG, as shown in Fig. 6. In this figure the DC versus $\mathrm{P}_{\lambda \mathrm{a}}$ curve has a resolution of about $4.399 \% / \mathrm{dB}$. The measure-

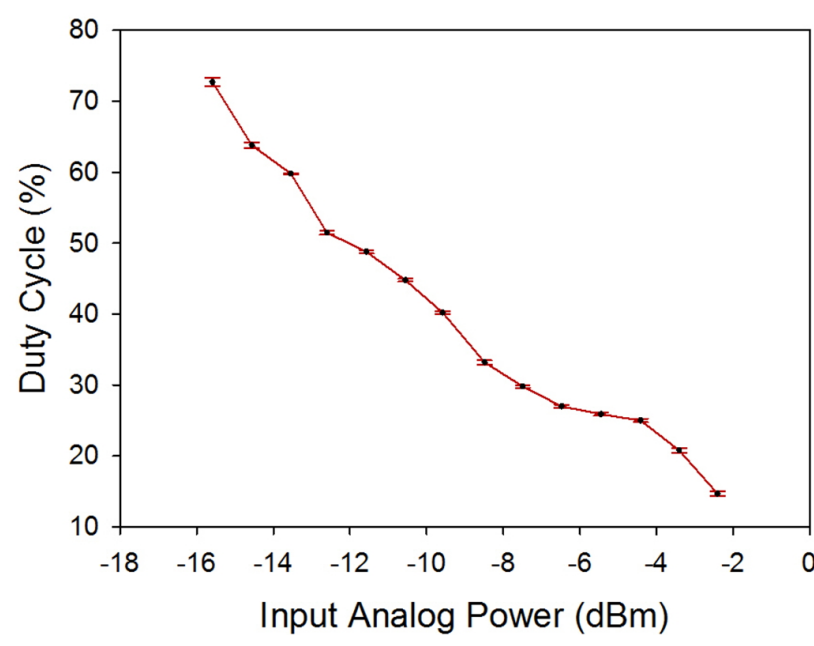

FIG. 6. Plot of the output PWM's duty cycle versus power $P_{\lambda}$ $a$ with standard error bars. The curve shows a resolution of about $4.399 \% / \mathrm{dB}$.

ment process is taken as follows: The value of each data point on the curve (represented by a black dot) was the average of 5 readings of the DC value (20-second interval) from the OSC. The error bars, which are included for every data point on the curve, reflected the standard error based on the recorded DC values. The reading error is seen to increase at low input power, which may arise from the increase in mode-partition noise of the SMFP-LD when the input analog power is low [17], and also from the mode beating between the injected and self-locked modes. The highest observed standard error was about $0.6 \%$ at input power of $-15.48 \mathrm{dBm}$. If the input power is above $-2.48 \mathrm{dBm}$, all modes of the single mode FP-LD are collapsed $(\mathrm{DC}=0)$. If the input power is below $-15.48 \mathrm{dBm}$, the locking state is unstable and the output signal so noisy that the output 
DC cannot be identified. When we decreased the input power about $1 \mathrm{~dB}$ more, the locking state was destroyed totally $(\mathrm{DC}=100 \%)$.

\section{CONCLUSION}

We have proposed and experimentally verified an optical PWM generator, which is based on the injection-locking property of an external cavity-based single-mode FP-LD. The OPWMG can directly generate a PWM signal in the optical domain with a working frequency of $16 \mathrm{MHz}$, a duty cycle range between $14.7 \%$ and $72.1 \%$, clear output waveforms, and duty cycle resolution of about $4.399(\% / \mathrm{dB})$. The PWM's duty cycle can be varied by tuning the power of the optical analog input. It is possible to increase the working frequency of the OPWMG up to several GHz, because the proposed scheme is based on injection-locking of an SMFP-LD. Indeed, several works have been based on injection-locking of SMFP-LD showing working frequencies up to $10 \mathrm{GHz}$, such as those are reported in $[15,16]$. An Optical PWM possesses significant advantages for applications that require strong immunity to EMI (e.g. in an HDVC plant) and an electrically isolated amplifier (e.g. in medical electronics).

\section{ACKNOWLEDGMENT}

The authors are deeply thankful to all interested persons at MKE and the Korea Evaluation Institute of Industrial Technology (KEIT).

\section{REFERENCES}

1. S. Suh, "Pulse width modulation for analog fiber-optic communications," IEEE J. Lightwave Technol. 5, 102-112 (1987).

2. Z. Liu and L. Jiang, "PWM speed control system of DC motor based on AT89S51," in Proc. 2011 International Conference on Electronic and Mechanical Engineering and Information Technology (EMEIT) (Harbin University, Harbin, Heilongjiang, China, Aug. 2011), vol. 3, pp. 1301-1303.

3. J. Flores-Arias, A. Moreno-Muoz, F. Domingo-Perez, V. Pallares-Lopez, and D. Gutierrez, "Voltage regulator system based on a PWM AC chopper converter," in Proc. IEEE International Symposium on Industrial Electronics (ISIE) (Gdansk University of Technology, Gdansk, Poland, Jun. 2011), pp. 468-473.
4. V. Tipsuwanporn, A. Charoen, A. Numsomran, and K. Phipek, "A single-phase PWM inverter controlling base on PLL technique," in Proc. SICE Annual Conference (SICE) (Waseda University, Tokyo, Japan, Sep. 2011), pp. 1178-1183.

5. J. Lindstrand, D. Radjen, R. Fitzgerald, A. Axholt, and H. Sjoland, "An integrated 3-level fully adjustable PWM class-D audio amplifier in $0.35 \mu \mathrm{m}$ CMOS," in Proc. NORCHIP (Tallinn, Estonia, Nov. 2008), pp. 168-171.

6. B. Wilson and Z. Ghassemlooy, "Optical pulse width modulation for electrically isolated analogue transmission," J. Phys. E: Sci. Instrum. 18, 954 (1985).

7. R. Perez, Design of Medical Electronic Devices (Academic Press, 2002).

8. R. McDowall, Fundamentals of HVAC Control Systems (Elsevier Science, Oxford, 2008).

9. B. E. Danielsson, "HVDC valve with light-triggered thyristors," in Proc. International Conference on AC and DC Power Transmission 1991 (London, UK, Sep. 1991), pp. 159-164.

10. D. Xianda, C. Englefield, P. Goud, and R. Razdan, "Four-level pulse width modulation for fiber optic communications," IEEE Transactions on Communications 30, 1958-1963 (1982).

11. Y.-I. Matsubara, J. Li, H. Yoshida, S. Shinohara, E. Tsuchiya, and H. Ikeda, "Pulse-width-modulated video signal transmission through optical fiber," in Proc. The 35th Midwest Symposium on Circuits and Systems (Washington D. C., USA, Aug. 1992), pp. 357-360.

12. Y. D. Jeong, Y. H. Won, S. O. Choi, and J. H. Yoon, "Tunable single-mode Fabry-Pérot laser diode using a built-in external cavity and its modulation characteristics," Opt. Lett. 31, 2586-2588 (2006).

13. Q. H. Tran, B. Nakarmi, and Y. H. Won, "An optical pulse width modulation generator based on the injection-locking property of single mode FP-LD," Proc. SPIE - Optical Components and Materials X 8621, 86210C (2013).

14. N. L. Hoang, J. S. Cho, Y. H. Won, and Y. D. Jeong, "Alloptical flip-flop with high on-off contrast ratio using two injection-locked single-mode Fabry-Pérot laser diodes," Opt. Express 15, 5166-5171 (2007).

15. B. Nakarmi, M. Rakib-Uddin, and Y. H. Won, "Realization of all-optical multi-logic functions and a digital adder with input beam power management for multi-input injection locking in a single-mode Fabry-Pérot laser diode," Opt. Express 19, 14121-14129 (2011).

16. H. T. Quoc, B. Nakarmi, and Y. H. Won, "All-optical address decoder using injection-locking property of external cavity based single mode FP-LD," IEEE Photonics Journal 5, 7900811 (2013).

17. K. Iwashita and K. Nakagawa, "Suppression of mode partition noise by laser diode light injection," IEEE J. Quantum Electron. 18, 1669-1674 (1982). 